\title{
ACÚMULO DE NUTRIENTES EM FRUTOS DE CAFEEIRO EM QUATRO ALTITUDES DE CULTIVO: CÁLCIO, MAGNÉSIO E ENXOFRE ${ }^{(1)}$
}

\author{
Bruno Galvêas Laviola ${ }^{(2)}$, Herminia Emilia Prieto Martinez $^{(3)}$, Luiz \\ Carlos Chamhum Salomão( ${ }^{(3)}$, Cosme Damião Cruz ${ }^{(4)} \&$ Sebastião \\ Marcos Mendonça ${ }^{(5)}$
}

\begin{abstract}
RESUMO
As curvas de acúmulo de nutrientes em frutos permitem estimar sua exportação, bem como as épocas de maior demanda. Estudou-se o acúmulo de MS, $\mathrm{Ca}, \mathrm{Mg}$ e $\mathrm{S}$ em frutos de Coffea arabica (L.) da antese à maturação em quatro altitudes, bem como a variação na concentração dos elementos em folhas dos ramos produtivos. O experimento foi constituído da variedade de cafeeiro Catuaí IAC 44 cultivada a 720, 800, 880 e 950 m de altitude, no município de Martins Soares-MG. O delineamento experimental foi inteiramente ao acaso, com três repetições, usando-se um esquema de parcela subdividida no tempo. $O$ aumento da altitude influenciou o ciclo reprodutivo do cafeeiro, pois houve demanda de maior tempo para formação dos frutos. No estádio de expansão rápida, a percentagem de acúmulo de MS, Ca, Mg e S foi maior na altitude de 720 m, comparada, principalmente, à de 950 m. A TMAD (taxa máxima de acúmulo diário) no estádio de granação-maturação apresentou tendência de ser mais tardia com a elevação da altitude. $O$ consumo de nutrientes pelos frutos, assim como o enchimento de grãos, é mais crítico em condições de menor altitude, já que a planta necessita completar esses processos em menor espaço de tempo. De modo geral, na altitude de $720 \mathrm{~m}$ ocorreu maior competição fruto/folha pela partição de Ca, $\mathrm{Mg}$ e S.
\end{abstract}

Termos de indexação: fisiologia vegetal, nutrição mineral, temperatura.

\footnotetext{
${ }^{(1)}$ Parte da Tese de Doutorado em Fitotecnia do primeiro autor. Recebido para publicação em novembro de 2006 e aprovado em julho de 2007.

${ }^{(2)}$ Engenheiro-Agrônomo, DSc., Pesquisador, Embrapa Agroenergia, CEP 70770-901 Brasília (DF). E-mail: bruno.laviola@embrapa.br

${ }^{(3)}$ Professor(a) do Departamento de Fitotecnia, Universidade Federal de Viçosa - UFV. CEP 36570-000 Viçosa (MG). E-mails: herminia@ufv.br; lsalomao@ufv.br

(4) Professor do Departamento de Biologia Geral, UFV. Email: cdcruz@ufv.br

${ }^{(5)}$ Engenheiro-Agrônomo, MS, Pesquisador do Centro de Pesquisas Cafeeiras Eloy Carlos Heringer - CEPEC. CEP 36900-000 Manhuaçu (MG). E-mail: sebastiao.mendonca@heringer.com.br
} 


\title{
SUMMARY: NUTRIENT ACCUMULATION IN COFFEE FRUITS AT FOUR PLANTATIONS ALTITUDE: CALCIUM, MAGNESIUM AND SULFUR
}

\begin{abstract}
Crurves of fruit nutrient accumulation are tools for estimating the peaks of nutrient demand and the nutrient export form the soil. The accumulation of dry matter (DM), Ca, $M g$ and $S$ was studied in fruits of Coffea arabica $(L$.$) in the period between anthesis and$ maturation at four altitudes of cultivation, as well as the variation in the leaf content of the elements in productive branches. The experiment consisted of the coffee variety Catuaí IAC 44 cultivated at 720, 800, 880 and $950 \mathrm{~m}$ above sea level (asl), in Martins Soares-MG. The experimental design was entirely randomized with tree replications using a split-plot in time scheme. The height asl of the crop influenced the coffee reproduction cycle, particularly the time required for fruit formation. In the fast expansion stage the percentages of DM and $\mathrm{Ca}, \mathrm{Mg}$ and $S$ accumulation were higher in trees grown at $720 \mathrm{~m}$ than at $950 \mathrm{~m}$. The DMAR (daily maximum accumulation rate) in the grain filling stage tended to be delayed at higher altitudes. Nutrient accumulation in the fruits as well as grain filling are more critical at lower altitudes since these processes have to occur in a shorter time. In general, the fruit/leaf competition for the partitioning of Ca, $\mathrm{Mg}$ and $S$ was higher at $720 \mathrm{~m}$.
\end{abstract}

Index terms: plant physiology; mineral nutrition; temperature.

\section{INTRODUÇÃ̃O}

O cafeeiro é uma planta que leva dois anos para completar o ciclo fenológico. No primeiro ano do ciclo, durante os meses de dias longos, ocorre a formação do corpo vegetativo da planta, com gemas axilares que poderão ser vegetativas ou reprodutivas, dependendo do estímulo recebido (Rena \& Maestri, 1985; Gouveia, 1984). O segundo ano do ciclo fenológico do cafeeiro começa com a floração, após um choque hídrico nas gemas florais. Após a fecundação da flor, inicia-se o período de desenvolvimento do fruto, entre os meses de setembro e julho, passando pelos estádios de chumbinho, expansão rápida, granação até a maturação (Rena et al., 2001; Camargo \& Camargo, 2001). As fases fenológicas do cafeeiro são bem definidas, porém, segundo Camargo (1998), elas podem adiantar ou atrasar, dependendo do clima e da região, além da altitude.

O crescimento do fruto do café em tamanho e matéria fresca segue um modelo-padrão de sigmóide dupla, embora um modelo sigmóide simples já tenha sido relatado (Rena \& Maestri, 1985). Laviola et al. (2007) verificou forte acúmulo de matéria seca no fruto nos estádios de expansão rápida e granação-maturação; neste último estádio, o acúmulo de matéria seca pelo fruto perdurou até o momento da colheita.

Durante a formação do fruto do cafeeiro e nos diversos estádios de desenvolvimento, há variações na concentração e na quantidade dos elementos acumulados, assim como na produção de matéria seca. De acordo com Moraes \& Catani (1964), o consumo de nutrientes e o acúmulo de matéria seca são intensificados a partir do quarto mês após a floração. Segundo Matiello et al. (2005), a quantidade de nutrientes exigida na fase de florada e chumbinho é pequena, aumentando significativamente a partir da passagem dos frutos para o estádio verde-aquoso, na granação (verde-sólido), até a maturação dos frutos. Cerca de $73 \%$ do crescimento vegetativo ocorre de outubro a abril, sendo o consumo de nutrientes para frutificação também concentrado nesse período (mais de $80 \%$ ).

As curvas de acúmulo de nutrientes em frutos de cafeeiro durante o período reprodutivo são uma importante ferramenta para estimar as necessidades nutricionais da cultura, bem como identificar os momentos mais adequados para aplicação de fertilizantes (Ramirez et al., 2002).

No Brasil, geralmente, o cafeeiro vem sendo cultivado em regiões com altitudes que variam de 400 a 1.200 m (Matiello et al., 2005). Em regiões de maior altitude, a planta de cafeeiro leva maior tempo para completar o ciclo reprodutivo. A influência da altitude no ciclo reprodutivo do cafeeiro está relacionada, principalmente, às temperaturas mais amenas em maiores altitudes.

É possível que o pico de exigência nutricional em cafeeiros plantados em regiões de altitude mais elevada seja mais tardio que em regiões de baixas altitudes. Dessa forma, as épocas e os intervalos entre as práticas de adubação deveriam ser diferenciados, levando-se em conta o período de maior exigência da planta de cafeeiro em cada região.

Foi objetivo deste trabalho o estudo do acúmulo de $\mathrm{Ca}, \mathrm{Mg}$ e S em frutos de cafeeiro arábico da antese à maturação, bem como a variação da concentração dos elementos em folhas dos ramos produtivos, em quatro altitudes de implantação da lavoura. 


\section{MATERIAL E MÉTODOS}

O experimento foi realizado durante o período de setembro de 2005 a julho de 2006, no Centro de Pesquisas Cafeeiras Eloy Carlos Heringer (CEPEC), localizado no município de Martins Soares, MG. O experimento foi constituído da variedade de Coffea arabica L. Catuaí IAC-44 em quatro altitudes (Quadro 1).

No mês de agosto de 2005 foi realizada a análise química do solo de cada talhão da propriedade (Quadro 2), para efetuar a correção de acidez do solo e o fornecimento de nutrientes minerais. $\mathrm{O}$ fornecimento de macronutrientes foi feito via solo, em função da fertilidade deste e da carga pendente de frutos (Guimarães et al., 1999) (Quadro 3). O enxofre foi fornecido como elemento acompanhante de fertilizantes nitrogenados. Os micronutrientes $\mathrm{Zn}, \mathrm{B}$ e $\mathrm{Cu}$ foram supridos por meio de três aplicações foliares anuais
(19/10/2005, 22/12/2005 e 22/01/2006), utilizando-se sulfato de zinco, ácido bórico, hidróxido de cobre e cloreto de potássio (como adjuvante), na concentração de $4 \mathrm{~g} \mathrm{~L}^{-1}$ de cada fertilizante.

$\mathrm{O}$ delineamento experimental empregado foi inteiramente ao acaso, distribuído em um esquema de parcelas subdivididas no tempo, com quatro parcelas (altitudes), 12 subparcelas (datas de amostragens) e três repetições. Para cada altitude foram selecionadas 20 plantas, dispostas em quatro fileiras, que constituíram as parcelas experimentais.

As amostragens iniciaram-se em 11 de outubro de 2005, quando houve antese floral, ocasião em que se coletaram folhas e flores, sendo este considerado como dia zero. A partir dessa data efetuaram-se coletas periódicas de folhas e frutos durante o desenvolvimento reprodutivo do cafeeiro, aos $34,52,65,85,100,114$, $134,161,190,217,239$ e 266 dias após a antese (DAA).

Quadro 1. Caracterização dos sítios (altitude) estudadas

\begin{tabular}{cccc}
\hline Altitude & Idade & Espaçamento & \\
\hline $\mathrm{m}$ & & $\mathrm{m}$ & Produtividade $^{(2)}$ \\
720 & 4 anos ${ }^{-1}$ & $2,0 \times 1,0$ & 31,60 \\
800 & 10 anos & $2,0 \times 1,0$ & 51,40 \\
880 & 10 anos & $2,0 \times 1,0$ & 55,60 \\
950 & 11 anos & $1,7 \times 0,7$ & 45,16
\end{tabular}

${ }^{(1)}$ Lavoura recepada em $2002 .{ }^{(2)}$ Uma saca de café pesa $60 \mathrm{~kg}$.

Quadro 2. Características químicas e físicas dos solos da área experimental em agosto de 2005

\begin{tabular}{|c|c|c|c|c|c|c|c|c|}
\hline Altitude & MO & $\mathrm{pH} \mathrm{H}_{2} \mathrm{O}$ & $\mathbf{P}$ & $\mathbf{K}$ & $\mathrm{Ca}^{2+}$ & $\mathrm{Mg}^{2+}$ & $\mathrm{Al}^{3+}$ & $\mathrm{H}+\mathrm{Al}$ \\
\hline $\mathrm{m}$ & \multicolumn{2}{|l|}{ dag $\mathrm{kg}^{-1}$} & \multicolumn{2}{|c|}{$-\mathrm{mg} \mathrm{dm}^{-3}$} & \multicolumn{3}{|c|}{$-\mathrm{cmol}_{\mathrm{c}} \mathrm{dm}^{-3}$} & + \\
\hline 720 & 3,58 & 6,1 & 27,0 & 132 & 4,2 & 0,9 & 0 & 4,29 \\
\hline 800 & 3,58 & 5,9 & 18,9 & 195 & 4,6 & 0,8 & 0 & 5,45 \\
\hline 880 & 3,20 & 5,0 & 21,1 & 117 & 2,2 & 0,3 & 0 & 7,43 \\
\hline \multirow[t]{3}{*}{950} & 4,61 & 5,2 & 7,5 & 116 & 2,4 & 0,3 & 0 & 8,25 \\
\hline & CTC & $\mathrm{S}$ & V & $\mathrm{Zn}$ & $\mathrm{Fe}$ & Mn & $\mathbf{C u}$ & B \\
\hline & $\mathrm{cmol}_{\mathrm{c}} \mathrm{dm}^{-3}$ & $\mathrm{mg} \mathrm{dm} \mathrm{m}^{-3}$ & $\%$ & $\ldots$ & 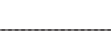 & $\mathrm{mg} \mathrm{dm}$ & & 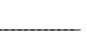 \\
\hline 720 & 9,73 & 22,46 & 56 & 27,1 & 104,0 & 45,3 & 3,9 & 1,54 \\
\hline 800 & 11,35 & 32,39 & 52 & 34,9 & 68,7 & 26,4 & 6,6 & 4,03 \\
\hline 880 & 10,23 & 36,54 & 27 & 20,9 & 77,5 & 32,3 & 6,4 & 1,83 \\
\hline 950 & 11,25 & 24,13 & 27 & 13,5 & 28,1 & 12,4 & 3,1 & 1,54 \\
\hline
\end{tabular}

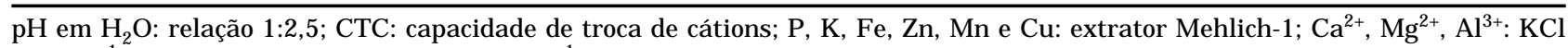
$1 \mathrm{~mol} \mathrm{~L}{ }^{-1} ; \mathrm{H}+\mathrm{Al}$ : método $\mathrm{Ca}(\mathrm{OAc})_{2} 0,5 \mathrm{~mol} \mathrm{~L}^{-1} ; \mathrm{pH} 7$. 
Quadro 3. Adubação empregada no ano agrícola de 2005/2006

\begin{tabular}{cccc}
\hline Altitude & $\mathbf{N}$ & $\mathbf{P}_{2} \mathbf{O}_{5}$ & $\mathbf{K}_{2} \mathbf{O}$ \\
\hline $\mathrm{m}$ & & $\mathrm{kg} \mathrm{ha}^{-1}$ & \\
720 & 390 & 0 & 200 \\
800 & 400 & 0 & 150 \\
880 & 400 & 0 & 150 \\
950 & 370 & 0 & 70 \\
\hline
\end{tabular}

Os frutos foram colhidos aleatoriamente na parcela de ramos pertencentes ao terço médio da planta - e as folhas foram correspondentes aos terceiro e quarto pares, contadas a partir do ápice de ramos com frutos, também situados no terço médio da planta. Foram coletados 100 frutos/parcela nas quatro primeiras amostragens, 60 frutos/parcela entre a quinta e a oitava amostragem e 20 frutos/parcela nas amostragens seguintes. As folhas foram coletadas em um número fixo de 15 folhas/parcela durante todas as amostragens.

Durante o período reprodutivo, as temperaturas mínimas e máximas, a umidade relativa (UR) entre 9 e 10 horas da manhã (Quadro 4) e o índice pluviométrico (Quadro 5) foram monitorados em intervalos de tempo semanais.

O material vegetal coletado foi lavado em água desionizada e seco em estufa de circulação de ar forçado a $70{ }^{\circ} \mathrm{C}$ até atingir peso constante, conforme descrito por Jones Junior et al. (1991). Após esse processo, o material vegetal foi pesado em balança de precisão, moído em moinho tipo Wiley, de aço inoxidável, passado em peneira de malha de $0,841 \mathrm{~mm}$ e acondicionado em embalagens de papel devidamente identificadas, para realização das análises químicas.

Para determinação dos teores de $\mathrm{Ca}, \mathrm{Mg}$ e S, o material vegetal, seco e moído, foi submetido à digestão nitroperclórica (Johnson \& Ulrich, 1959). O Ca e o
Quadro 5. Índice pluviométrico mensal durante o ano agrícola 2005/06 em Martins Soares, MG

\begin{tabular}{|c|c|c|c|c|c|c|}
\hline \multicolumn{7}{|c|}{ Ano 2005} \\
\hline \multirow{2}{*}{$\begin{array}{c}\text { Agosto } \\
12,0\end{array}$} & \multirow{2}{*}{$\begin{array}{c}\text { Setembro } \\
168,4\end{array}$} & \multirow{2}{*}{$\begin{array}{c}\text { Outubro } \\
59,3\end{array}$} & \multicolumn{2}{|c|}{ o Novembro } & \multicolumn{2}{|c|}{ Dezembro } \\
\hline & & & 3 & 53,5 & 278 & \\
\hline \multicolumn{7}{|c|}{ Ano 2006} \\
\hline Janeiro & Fevereiro & Março & Abril & Maio & Junho & Julho \\
\hline 7,0 & 131,0 & 256,5 & 121,8 & 31,0 & 8,0 & 13,8 \\
\hline
\end{tabular}

$\mathrm{Mg}$ foram quantificados por espectrofotometria de absorção atômica (AOAC, 1975), enquanto o S foi avaliado por turbidimetria do sulfato (Jackson, 1958).

O acúmulo dos nutrientes por fruto ( $\mathrm{mg} /$ fruto) foi calculado pela seguinte fórmula:

Acúmulo $=\frac{\text { MS de fruto }(\mathrm{mg}) \times \text { concentração de nutriente }\left(\text { dag kg }^{-1}\right)}{100}$

Os dados obtidos foram submetidos à análise de variância e regressão. Para explicar fisiologicamente o acúmulo de MS, $\mathrm{Ca}, \mathrm{Mg}$ e $\mathrm{S}$ em frutos e a variação de nutrientes em folhas, utilizaram-se modelos de regressões não-lineares sigmoidais com três e quatro parâmetros e cúbico, conforme descrito a seguir. A escolha dos modelos foi de acordo com o ajuste $\left(R^{2}\right)$ e a melhor representação do fenômeno.

$$
\begin{aligned}
& \hat{y}=\frac{a}{1+\exp -\left(\frac{x-x_{0}}{b}\right)} \\
& \hat{y}=y_{0}+\frac{a}{1+\exp -\left(\frac{x-x_{0}}{b}\right)} \\
& a=\text { ponto de máximo da curva } \quad a=\mathrm{y}_{\max }-\mathrm{y}_{\min } \\
& b=\text { parâmetro de ajuste } \quad b=\text { parâmetro de ajuste } \\
& x_{0}=\text { ponto de inflexão } \quad x_{0}=\text { ponto de inflexão } \\
& y_{0}=\text { ponto de mínimo da curva } \\
& \hat{y}=y_{0}+a x+b x^{2}+c x^{3}
\end{aligned}
$$

\begin{tabular}{|c|c|c|c|c|}
\hline \multirow{2}{*}{ Temperatura } & \multicolumn{4}{|c|}{ Altitude } \\
\hline & $720 \mathrm{~m}$ & $800 \mathrm{~m}$ & $880 \mathrm{~m}$ & $950 \mathrm{~m}$ \\
\hline Mínima média $\left({ }^{\circ} \mathrm{C}\right)$ & 16,22 & 17,28 & 18,94 & 17,33 \\
\hline Máxima média $\left({ }^{\circ} \mathrm{C}\right)$ & 30,22 & 29,89 & 29,50 & 26,94 \\
\hline Umidade relativa (\%) & 73,33 & 77,50 & 65,67 & 81,50 \\
\hline
\end{tabular}

Quadro 4. Temperaturas mínimas e máximas e umidade relativa (UR) médias ocorridas durante o período reprodutivo do cafeeiro, em quatro altitudes 
Os pontos de inflexão das curvas ajustadas corresponderam aos momentos em que ocorreram as taxas máximas de acúmulo de matéria seca e nutrientes em frutos. A taxa máxima de acúmulo diário (TMAD, mg/dia) foi determinada pelo acúmulo de matéria seca e nutrientes no ponto de inflexão menos o acúmulo do dia anterior.

Os pontos de curvatura mínima $\left(\mathrm{PC}_{\min }\right)$ e máxima $\left(\mathrm{PC}_{\max }\right)$ nos modelos sigmoidais foram calculadas conforme o método citado por Venegas et al. (1998), utilizando os parâmetros das equações não-lineares:

$$
C_{\text {min }}=x_{0}-2 b \quad C_{\text {máx }}=x_{0}+2 b
$$

$\mathrm{O} \mathrm{PC}_{\min }$ indica o momento na curva de acúmulo em que se iniciam ganhos significativos no acúmulo de MS, Ca, Mg e S. Já o $\mathrm{PC}_{\max }$ indica o momento em que 0 acúmulo dos elementos começa a se estabilizar.

$\mathrm{O}$ acúmulo relativo (ARe) foi obtido de acordo com as diferenças entre o mínimo e o máximo acúmulo em cada estádio de formação do fruto, sendo os valores em percentagem obtidos em relação ao acúmulo final alcançado.

$$
\text { ARe }=A_{F}-A_{I}
$$

$A R e=$ acúmulo relativo no estádio de formação do fruto

$A_{F}$ e $A_{I}=$ acúmulo no final e início do estádio

$$
\%=\frac{A R e}{A T} * 100
$$

$\mathrm{AT}=$ acúmulo alcançado aos 266 DAA
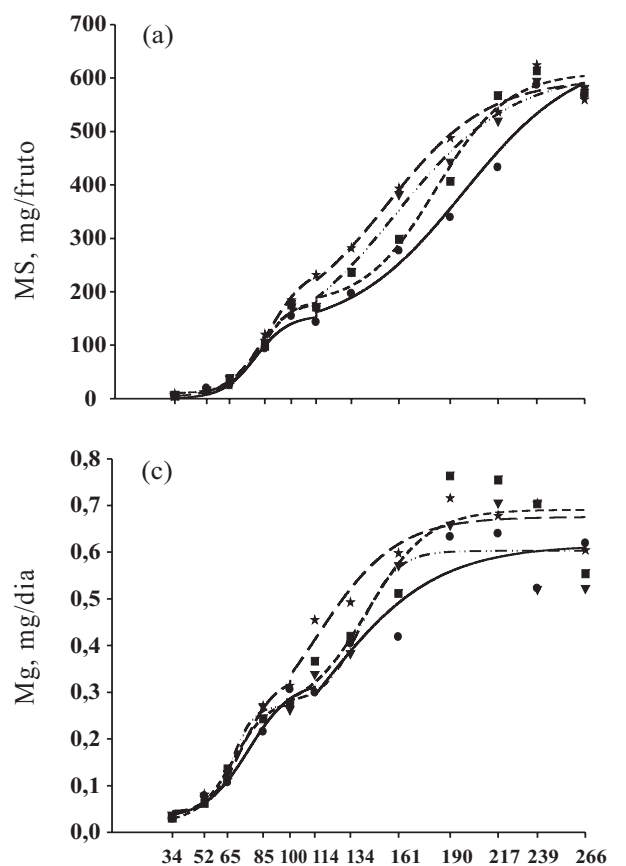

Calculou-se a diferença das concentrações foliares no início do ciclo reprodutivo, na época da floração $\left(\mathrm{C}_{\mathrm{F}}\right)$, em relação às concentrações foliares de $\mathrm{Ca}, \mathrm{Mg}$ e $\mathrm{S}$ nos respectivos momentos TMAD (taxa máxima de acúmulo diário) para os frutos nos estádios de expansão rápida $\left(\mathrm{C}_{\mathrm{ER}}\right)$ e granação $\left(\mathrm{C}_{\mathrm{G}}\right)$. Esse gradiente de concentração indica se houve competição fruto/folha pelo acúmulo de $\mathrm{Ca}, \mathrm{Mg}$ e $\mathrm{S}$ nos momentos de maior exigência nutricional da cultura.

\section{RESULTADOS E DISCUSSÃO}

Com base nas curvas de acúmulo de matéria seca (Figura 1a; Quadro 6), foi possível distinguir os quatro estádios de formação dos frutos, sendo eles, de acordo com Rena et al. (2001), denominados de chumbinho, expansão rápida, crescimento suspenso granação e maturação (Quadro 7).

No estádio de chumbinho, os frutos apresentam baixa taxa de crescimento (acúmulo de matéria seca), porém elevada taxa respiratória e de multiplicação celular (Leon \& Fournier, 1962; Cannel, 1971a). Esse estádio apresentou duração variando entre 61 e 67 DAA (11 a 16/12/2005) (Quadro 7). Não se observou neste trabalho efeito do aumento crescente da altitude no acúmulo de $\mathrm{MS}, \mathrm{Ca}, \mathrm{Mg}$ e $\mathrm{S}$ em frutos de cafeeiro no estádio de chumbinho (Figura 1; Quadros 6, 7 e 8). É possível que os efeitos da altitude, principalmente os relacionados à temperatura (Quadro 4), influenciem menos nas primeiras semanas de formação do fruto de cafeeiro.
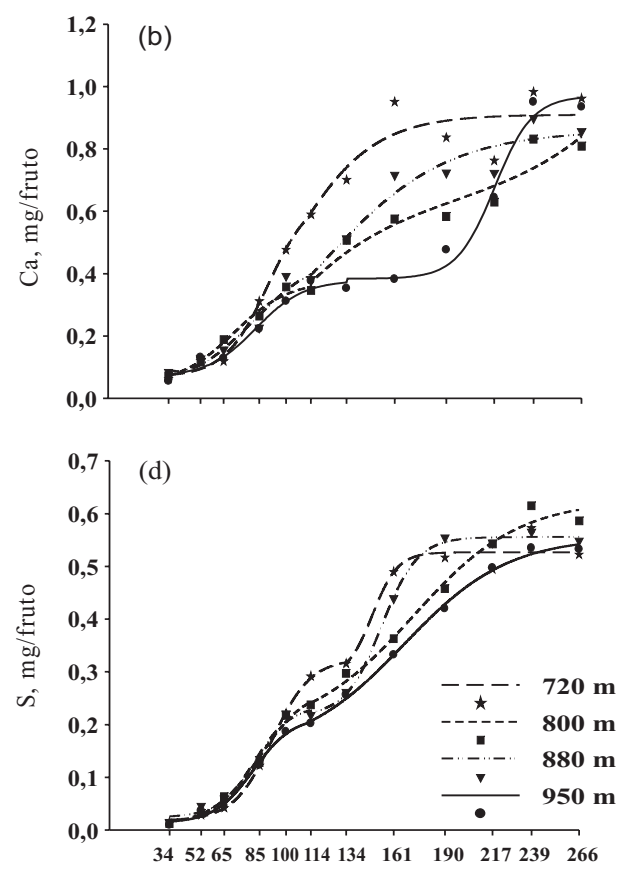

DIAS APÓS A ANTESE

Figura 1. Acúmulo de MS (A), Ca (B), Mg (C) e S (D) em frutos de cafeeiro em função do tempo decorrido após $a$ antese, em quatro altitudes. 
Quadro 6. Equações de regressão do acúmulo de MS e Ca, Mg e S em frutos de cafeeiros em função do tempo decorrido após a antese

\begin{tabular}{|c|c|c|c|c|}
\hline Variável & Altitude & Período & Modelo & $\mathbf{R}^{2}$ \\
\hline \multirow[t]{8}{*}{ MS } & $720 \mathrm{~m}$ & $34-114$ & $\hat{y}=3,0983+246,5243 /(1+\exp (-(x-87,2082) / 11,1720))$ & 0,997 \\
\hline & & $100-266$ & $\hat{y}=131,5992+466,9270 /(1+\exp (-(x-154,5708) / 28,4426))$ & 0,980 \\
\hline & $800 \mathrm{~m}$ & $34-114$ & $\hat{y}=10,1586+174,0190 /(1+\exp (-(x-83,1578) / 8,6302))$ & 0,986 \\
\hline & & $100-266$ & $\hat{y}=173,9505+436,6964 /(1+\exp (-(\mathrm{x}-181,8763) / 20,2356))$ & 0,982 \\
\hline & $880 \mathrm{~m}$ & $34-114$ & $\hat{y}=9,5054+170,4282 /(1+\exp (-(\mathrm{x}-81,2099) / 8,9412))$ & 0,991 \\
\hline & & $100-266$ & $\hat{y}=80,5608+530,4816 /(1+\exp (-(\mathrm{x}-159,0731) / 32,9222$ & 0,986 \\
\hline & $950 \mathrm{~m}$ & $34-114$ & $\hat{y}=156,1461 /(1+\exp (-(\mathrm{x}-79,7382) / 9,3653))$ & 0,977 \\
\hline & & $100-266$ & $\hat{y}=124,7881+521,8913 /(1+\exp (-(\mathrm{x}-197,4214) / 32,4306))$ & 0,974 \\
\hline \multirow[t]{8}{*}{$\mathrm{Ca}$} & $720 \mathrm{~m}$ & $34-114$ & $\hat{y}=0,06881+0,59275 /(1+\exp (-(\mathrm{x}-90,0026) / 12,2515))$ & 0,994 \\
\hline & & $100-266$ & $\hat{y}=0,91010 /(1+\exp (-(\mathrm{x}-98,8445) / 23,8213))$ & 0,819 \\
\hline & $800 \mathrm{~m}$ & $34-114$ & $\hat{y}=0,04441+0,33211 /(1+\exp (\mathrm{x}-70,7687) / 15,3902))$ & 0,984 \\
\hline & & $114-266$ & $\hat{y}=-1,09827+0,02244 x-0,00010 x^{2}+0,0000001764 x^{3}$ & 0,903 \\
\hline & $880 \mathrm{~m}$ & $34-114$ & $\hat{y}=0,35062-0,01505 x+0,00025 x^{2}-0,000001033 x^{3}$ & 0,948 \\
\hline & & $114-266$ & $\hat{y}=0,85902 /(1+\exp (-(\mathrm{x}-119,5827) / 34,9757))$ & 0,916 \\
\hline & $950 \mathrm{~m}$ & $34-134$ & $\hat{y}=0,06983+0,30843 /(1+\exp (-(\mathrm{x}-83,0292) / 13,3889))$ & 0,970 \\
\hline & & $134-266$ & $\hat{y}=0,38364+0,58624 /(1+\exp (-(\mathrm{x}-217,1613) / 10,5631))$ & 0,979 \\
\hline \multirow[t]{8}{*}{$\mathrm{Mg}$} & $720 \mathrm{~m}$ & $34-100$ & $\hat{y}=0,03950+0,29368 /(1+\exp (-(x-73,7871) / 9,3143))$ & 0,992 \\
\hline & & $85-266$ & $\hat{y}=0,16535+0,51320 /(1+\exp (-(\mathrm{x}-115,0658) / 22,3578))$ & 0,941 \\
\hline & $800 \mathrm{~m}$ & $34-100$ & $\hat{y}=0,02124+0,26567 /(1+\exp (-(\mathrm{x}-68,0748) / 9,9950))$ & 0,999 \\
\hline & & $85-266$ & $\hat{y}=0,25345+0,43761 /(1+\exp (\mathrm{x}-143,2402) / 17,2493))$ & 0,872 \\
\hline & $880 \mathrm{~m}$ & $34-100$ & $\hat{y}=0,04449+0,22694 /(1+\exp (-(\mathrm{x}-67,9556) / 5,9365))$ & 0,991 \\
\hline & & $85-266$ & $\hat{y}=0,27644+0,32663 /(1+\exp (-(\mathrm{x}-139,5044) / 10,2302))$ & 0,864 \\
\hline & $950 \mathrm{~m}$ & $34-114$ & $\hat{y}=0,02946+0,29486 /(1+\exp (-(\mathrm{x}-76,7951) / 12,2022))$ & 0,986 \\
\hline & & $114-266$ & $\hat{y}=0,61541 /(1+\exp (-(\mathrm{x}-116,9673) / 31,9635))$ & 0,813 \\
\hline \multirow[t]{8}{*}{$\mathrm{S}$} & $720 \mathrm{~m}$ & $34-134$ & $\hat{y}=0,01830+0,30604 /(1+\exp (-(\mathrm{x}-92,2337) / 10,8988))$ & 0,999 \\
\hline & & $114-266$ & $\hat{y}=0,28949+0,23722 /(1+\exp (-(\mathrm{x}-148,9584) / 7,2383))$ & 0,954 \\
\hline & $800 \mathrm{~m}$ & $34-114$ & $\hat{y}=0,01102+0,25825 /(1+\exp (-(\mathrm{x}-85,2558) / 13,2537))$ & 0,992 \\
\hline & & $100-266$ & $\hat{y}=0,18558+0,44011 /(1+\exp (-(\mathrm{x}-172,046) / 29,9914))$ & 0,989 \\
\hline & $880 \mathrm{~m}$ & $34-114$ & $\hat{y}=0,02434+0,21190 /(1+\exp (-(\mathrm{x}-82,1982) / 10,1618))$ & 0,982 \\
\hline & & $100-266$ & $\hat{y}=0,21665+0,33904 /(1+\exp (-(\mathrm{x}-154,0678) / 10,2236))$ & 0,998 \\
\hline & $950 \mathrm{~m}$ & $34-114$ & $\hat{y}=0,01311+0,20181 /(1+\exp (-(\mathrm{x}-81,5785) / 11,1354))$ & 0,994 \\
\hline & & $100-226$ & $\hat{y}=0,14762+0,40801 /(1+\exp (-(\mathrm{x}-166,669) / 29,5987))$ & 0,997 \\
\hline
\end{tabular}

Pode-se observar que no estádio de chumbinho a proporção de acúmulo de Ca e Mg foi maior comparada à de acúmulo de MS e S (Quadro 9). É provável que o maior acúmulo de Ca nesse estádio esteja relacionado à grande importância deste nutriente nos processos de divisão celular e na estabilização de membranas e paredes celulares das novas células formadas (Marschner, 1995; Marenco \& Lopes, 2005). Com 
Quadro 7. Delimitação dos estádios de desenvolvimento do fruto de cafeeiro (DAA) durante o período reprodutivo com base nas curvas de acúmulo de matéria seca em frutos. Os valores entre parênteses representam a duração (dias) dos estádios

\begin{tabular}{|c|c|c|c|c|c|}
\hline Altitude & Chumbinho & Exp. rápida & Cresc. susp. & Granação & Maturação \\
\hline \multicolumn{6}{|l|}{$\mathrm{m}$} \\
\hline 720 & $0-65(65)$ & $65-109(44)$ & $109-114(5)$ & $114-211(97)$ & $211-266(55)$ \\
\hline 800 & $0-67(67)$ & $67-100(33)$ & $100-114(14)$ & $114-222(108)$ & $222-266(44)$ \\
\hline 880 & $0-63(63)$ & $63-99(36)$ & $99-114(15)$ & $114-224(110)$ & $224-266(42)$ \\
\hline 950 & $0-61(61)$ & $61-98(37)$ & $98-114(17)$ & $114-262(148)$ & $262-266(4)$ \\
\hline
\end{tabular}

Quadro 8. Ponto da taxa máxima de acúmulo diário ( $\left.\mathrm{X}_{\mathrm{TMAD}}, \mathrm{DAA}\right)$, taxa máxima de acúmulo diário (TMAD, $\mathrm{mg} /$ fruto/dia) e pontos de curvatura mínima $\left(\mathrm{PC}_{\min }, \mathrm{DAA}\right)$ e máxima $\left(\mathrm{PC}_{\text {max }}, \mathrm{DAA}\right)$, em quatro altitudes

\begin{tabular}{|c|c|c|c|c|c|c|}
\hline Variável & Altitude & Período & $\mathbf{X}_{\text {TMAD }}$ & TMAD & $\mathbf{C}_{\min }$ & $\mathbf{C}_{\max }$ \\
\hline & $\mathrm{m}$ & $d$ & & & & \\
\hline \multirow[t]{8}{*}{ MS } & 720 & $34-114$ & 87 & 5,5110 & 65 & 109 \\
\hline & & $100-266$ & 154 & 4,1025 & 114 & 211 \\
\hline & 800 & $34-114$ & 83 & 5,0323 & 67 & 100 \\
\hline & & $100-266$ & 182 & 5,3944 & 141 & 222 \\
\hline & 880 & $34-114$ & 81 & 4,7844 & 63 & 99 \\
\hline & & $100-266$ & 159 & 4,0279 & 93 & 224 \\
\hline & 950 & $34-114$ & 80 & 4,1665 & 61 & 98 \\
\hline & & $100-266$ & 197 & 4,0222 & 132 & 262 \\
\hline \multirow[t]{8}{*}{$\mathrm{Ca}$} & 720 & $34-114$ & 90 & 0,0120 & 66 & 114 \\
\hline & & $100-266$ & 99 & 0,0095 & 114 & 146 \\
\hline & 800 & $34-114$ & 71 & 0,0054 & 40 & 102 \\
\hline & & $100-266$ & 266 & 0,0045 & 114 & 266 \\
\hline & 880 & $34-114$ & 82 & 0,0056 & 34 & 114 \\
\hline & & $100-266$ & 119 & 0,0061 & 50 & 190 \\
\hline & 950 & $34-114$ & 83 & 0,0057 & 34 & 114 \\
\hline & & $100-266$ & 217 & 0,0138 & 196 & 238 \\
\hline \multirow[t]{8}{*}{$\mathrm{Mg}$} & 720 & $34-114$ & 74 & 0,0079 & 55 & 92 \\
\hline & & $114-266$ & 115 & 0,0057 & 114 & 160 \\
\hline & 800 & $34-134$ & 68 & 0,0060 & 48 & 88 \\
\hline & & $114-266$ & 143 & 0,0063 & 114 & 178 \\
\hline & 880 & $34-114$ & 68 & 0,0095 & 56 & 80 \\
\hline & & $114-266$ & 139 & 0,0079 & 119 & 160 \\
\hline & 950 & $34-134$ & 77 & 0,0060 & 52 & 101 \\
\hline & & $134-266$ & 117 & 0,0048 & 134 & 181 \\
\hline \multirow[t]{8}{*}{$\mathrm{S}$} & 720 & $34-114$ & 92 & 0,0070 & 70 & 114 \\
\hline & & $100-266$ & 149 & 0,0081 & 134 & 163 \\
\hline & 800 & $34-114$ & 85 & 0,0049 & 59 & 112 \\
\hline & & $100-266$ & 172 & 0,0037 & 114 & 232 \\
\hline & 880 & $34-114$ & 82 & 0,0052 & 62 & 103 \\
\hline & & $100-266$ & 154 & 0,0082 & 134 & 175 \\
\hline & 950 & $34-114$ & 81 & 0,0045 & 59 & 104 \\
\hline & & $100-266$ & 167 & 0,0034 & 114 & 206 \\
\hline
\end{tabular}


relação a Mg, este pode ter sido requerido em maior quantidade para acelerar a atividade de ATPases (Marschner, 1995), já que o fruto no estádio de chumbinho possui alta taxa respiratória (Cannel, 1971b; Rena et al, 2001).

O estádio de expansão rápida do fruto apresentou tendência de ser menos extenso na menor altitude, com exceção para a altitude de $720 \mathrm{~m}$ (Figura 1a; Quadros 6 e 7). Na altitude de $800 \mathrm{~m}$ o estádio de expansão rápida ocorreu entre 67 e 100 DAA (17/12/ 2005 a 19/01/2006), enquanto a 950 m o mesmo estádio apresentou duração entre 61 e 98 DAA (11/12/2005 a 17/01/2006) (Quadro 7). O acúmulo de matéria seca no estádio de expansão rápida está relacionado, principalmente, à expansão celular, com deposição de material de parede (Coombe, 1976), sendo o fluxo de água para os frutos essencial para o processo de alongamento celular (Marenco \& Lopes, 2005). É plausível que o acúmulo de nutrientes nos frutos no estádio de expansão ocorra por fluxo em massa, decorrente das altas taxas de translocação de água para os frutos neste estádio (Ramirez et al., 2002), necessária para expansão celular (Coombe, 1976; Taiz \& Zeiger, 2004).

$\mathrm{O}$ ponto de curvatura mínima $\left(\mathrm{PC}_{\min }\right)$ indica o momento em que se iniciam acúmulos relativos expressivos nos frutos (Quadro 8). De modo geral, a diferença de tempo decorrido até os $\mathrm{PC}_{\min }$ no estádio de expansão rápida, nas diversas altitudes, foi pequena, indicando pouco efeito da altitude sobre o início da ascensão de translocação de nutrientes para os frutos. Com base nos valores de $\mathrm{PC}_{\min }$ (Quadro 8), é imprescindível que os nutrientes já estejam disponíveis para absorção pelo sistema radicular antes do início do estádio de expansão rápida (Quadro 7).

O tempo decorrido até a taxa máxima de acúmulo diário $\left(\mathrm{X}_{\mathrm{TMAD}}\right)$ de $\mathrm{MS}, \mathrm{Ca}, \mathrm{Mg}$ e $\mathrm{S}$ em frutos foi maior a $720 \mathrm{~m}$, comparado às outras altitudes (Quadro 8). $\mathrm{O}$ maior $\mathrm{X}_{\mathrm{TMAD}}$ na altitude de $720 \mathrm{~m}$ está relacionado ao alcance de maiores TMAD nesta altitude. É provável que as maiores temperaturas ocorridas na altitude de $720 \mathrm{~m}$ (Quadro 4) tenham proporcionado maior atividade metabólica (Larcher, 2004) nos frutos e, conseqüentemente, maior velocidade de acúmulo de MS e nutrientes.

Maiores acúmulos relativos (ARe) de MS, $\mathrm{Ca}, \mathrm{Mg}$ e S no estádio de expansão rápida do fruto foram observados na altitude de $720 \mathrm{~m}$, com relação às outras altitudes (Quadro 9). Em outras palavras, na altitude de $720 \mathrm{~m}$ houve, em proporção, maior consumo dos nutrientes, bem como de fotoassimilados. A maior TMAD nesta altitude (Quadro 8) contribuir para que houvesse maior acúmulo de nutrientes no final do estádio.

O estádio de crescimento suspenso apresentou maior duração à medida que se foi aumentando a altitude (Figura 1a; Quadros 6 e 7). É possível que a menor velocidade de crescimento neste estádio esteja relacionada à reciclagem e síntese de enzimas e compostos intermediários (Taiz \& Zeiger, 2004), antes empregados na síntese de polímeros de parede, para serem utilizados como precursores na síntese de compostos de reservas no estádio de granação.

O estádio de granação teve início aos 114 DAA (02/ 02/2006), apresentando duração até 211 DAA (10/05/ 2006) na altitude de 720 m e até 262 DAA (30/06/2006) na de $950 \mathrm{~m}$. Em outras palavras, o ciclo reprodutivo do cafeeiro foi maior com o aumento da altitude. E provável que a ocorrência de menores temperaturas máximas (Quadro 4) tenha influenciado as reações enzimáticas e o transporte de fotoassimilados (Larcher, 2004), ampliando o tempo de formação do fruto de cafeeiro.

No estádio de granação, também chamado de enchimento do endosperma, a matéria seca é depositada, principalmente, nas sementes (Rena et al., 2001). $\mathrm{A} \mathrm{X}_{\mathrm{TMAD}}$ no estádio de granação (Quadro 8) foi mais precoce na altitude de $720 \mathrm{~m}$, comparada principalmente à altitude de $950 \mathrm{~m}$. A $720 \mathrm{~m}$ de altitude a $\mathrm{X}_{\text {TMAD }}$ de MS ocorreu aos 154 DAA (06/02/2006); a de $\mathrm{Ca}$, aos $99 \mathrm{DAA}(12 / 12 / 2005)$; a de $\mathrm{Mg}$, aos $115 \mathrm{DAA}$ (28/12/2005); e a de S, aos 149 DAA (01/02/2006). Na altitude de $950 \mathrm{~m}$, a $\mathrm{X}_{\text {TMAD }}$ foi aos $197 \mathrm{DAA}(21 / 03 /$ 2006) para MS, aos 217 DAA para Ca (10/04/2006), aos 117 DAA (30/12/2006) para Mg e aos 167 DAA para S. Esses foram os momentos de maior exigência nutricional do cafeeiro no estádio de granação, nas suas respectivas altitudes. A deficiência desses elementos poderia comprometer a fase final de formação da semente, já que $\mathrm{Ca}, \mathrm{Mg}$ e $\mathrm{S}$ fazem parte de diversos processos enzimáticos e metabólicos (Marschner, 1995).

A estabilização $\left(\mathrm{PC}_{\max }\right)$ no acúmulo de $\mathrm{Ca}, \mathrm{Mg}$ e $\mathrm{S}$ no estádio de granação ocorreu em menor tempo na altitude de $720 \mathrm{~m}$, confrontada com as demais (Quadro 8). Além disso, o acúmulo de nutrientes estabilizou-se antes do acúmulo de MS em frutos, ou seja, primeiro são acumulados os nutrientes para depois completar os processos de formação das sementes. O consumo de nutrientes pelos frutos, assim como o enchimento de grãos, é mais crítico em condições de menor altitude, já que a planta necessita completar esses processos em menor espaço de tempo.

A percentagem de acúmulo relativo no estádio de granação (Quadro 9) foi maior nas maiores altitudes, comparada à menor $(720 \mathrm{~m})$. Isso ocorreu, principalmente, em razão de o acúmulo de MS e nutrientes na altitude de $720 \mathrm{~m}$ ter se antecipado nas primeiras fases de formação do fruto.

Após o estádio de granação iniciou-se o de maturação, que se caracteriza, principalmente, pelo aumento do teor de açúcares e por mudanças na coloração da casca do fruto (Puschmann, 1975; Rena et al., 2001). Apesar de no quadro 7 o estádio de maturação ter ido até 266 DAA (época da última amostragem), na altitude de $720 \mathrm{~m}$ a mudança na coloração da casca do fruto de verde para vermelho foi visivelmente em menor tempo. 
Quadro 9. Acúmulo relativo (ARe) de acordo com o estádio de formação do fruto de cafeeiro, em quatro altitudes

\begin{tabular}{|c|c|c|c|c|c|c|c|c|c|c|c|}
\hline \multirow{3}{*}{ Variável } & \multirow{3}{*}{$\frac{\text { Altitude }}{\mathrm{m}}$} & \multicolumn{10}{|c|}{ Ganho de acúmulo } \\
\hline & & \multicolumn{2}{|c|}{ Chumbinho } & \multicolumn{2}{|c|}{ Exp. rápida } & \multicolumn{2}{|c|}{ Cresc. susp. } & \multicolumn{2}{|c|}{ Granação } & \multicolumn{2}{|c|}{ Maturação ${ }^{(1)}$} \\
\hline & & $\mathrm{mg} /$ fruto & $\%$ & $\mathrm{mg} /$ fruto & $\%$ & mg/fruto & $\%$ & $\mathrm{mg} /$ fruto & $\%$ & $\mathrm{mg} /$ fruto & $\%$ \\
\hline \multirow[t]{4}{*}{ MS } & 720 & 32,8 & 5,56 & 186,3 & 31,58 & 10,15 & 1,72 & 312,9 & 53,1 & 47,34 & 8,03 \\
\hline & 800 & 33,35 & 5,52 & 129,2 & 21,39 & 16,9 & 2,8 & 378,4 & 62,65 & 46,12 & 7,64 \\
\hline & 880 & 29,17 & 4,93 & 130,3 & 22,03 & 16,25 & 2,75 & 370,5 & 62,68 & 44,96 & 7,61 \\
\hline & 950 & 18,6 & 3,15 & 118,1 & 20 & 15,52 & 2,63 & 431,8 & 73,12 & 6,491 & 1,1 \\
\hline \multirow[t]{4}{*}{$\mathrm{Ca}$} & 720 & 0,137 & 15,06 & 0,421 & 46,29 & 0,03 & 3,35 & 0,314 & 34,5 & 0,007 & 0,8 \\
\hline & 800 & 0,19 & 22,65 & 0,143 & 17,04 & 0,024 & 2,9 & 0,336 & 39,97 & 0,146 & 17,45 \\
\hline & 880 & 0,141 & 16,64 & 0,196 & 23,15 & 0,06 & 7,13 & 0,421 & 49,72 & 0,028 & 3,36 \\
\hline & 950 & 0,12 & 12,41 & 0,183 & 18,93 & 0,048 & 5 & 0,611 & 63,37 & 0,003 & 0,27 \\
\hline \multirow[t]{4}{*}{$\mathrm{Mg}$} & 720 & 0,122 & 18,04 & 0,262 & 38,89 & 0,028 & 4,22 & 0,256 & 37,89 & 0,006 & 0,94 \\
\hline & 800 & 0,147 & 21,27 & 0,13 & 18,75 & 0,045 & 6,5 & 0,365 & 52,87 & 0,004 & 0,6 \\
\hline & 880 & 0,114 & 18,93 & 0,157 & 26,04 & 0,03 & 5,01 & 0,302 & 49,99 & 0,001 & 0,02 \\
\hline & 950 & 0,093 & 15,24 & 0,187 & 30,73 & 0,031 & 5,05 & 0,298 & 48,86 & 0,001 & 0,1 \\
\hline \multirow[t]{4}{*}{$\mathrm{s}$} & 720 & 0,042 & 7,89 & 0,229 & 43,42 & 0,018 & 3,33 & 0,239 & 45,35 & 0,001 & 0,1 \\
\hline & 800 & 0,063 & 10,38 & 0,142 & 23,44 & 0,037 & 6,16 & 0,313 & 51,53 & 0,052 & 8,5 \\
\hline & 880 & 0,052 & 9,38 & 0,15 & 27 & 0,025 & 4,53 & 0,328 & 59 & 0,001 & 0,06 \\
\hline & 950 & 0,041 & 7,49 & 0,137 & 25,24 & 0,027 & 5,01 & 0,335 & 61,9 & 0,02 & 0,35 \\
\hline
\end{tabular}

${ }^{(1)}$ Considerou-se como maturação o período após o início da estabilização no acúmulo de MS no fruto, embora as mudanças na coloração da casca correspondentes a maturação tenham iniciado antes.

O efeito da altitude no acúmulo de matéria seca e de $\mathrm{Ca}, \mathrm{Mg}$ e S está relacionado, sobretudo, à ocorrência de menores temperaturas máximas nas maiores altitudes (Quadro 4). De acordo com Larcher (2004), a temperatura tem influência direta sobre processos regulatórios da planta. Sob menores temperaturas, a velocidade das reações enzimáticas é reduzida e, conseqüentemente, as taxas fotossintéticas e respiratórias também são restringidas.

A ocorrência de temperaturas amenas desfavorece a absorção de íons pelas raízes. A elevação da temperatura aumenta a velocidade de difusão e de fluxo em massa de íons para as raízes, bem como a penetração de íons no espaço livre aparente via apoplasto, estimula a absorção e o acúmulo de íons nas células das raízes e favorece o transporte nos condutos do xilema. Além disso, o aumento da temperatura acelera a atividade respiratória da planta e incrementa o metabolismo nas raízes e a produção de ATP, liberando energia, que é utilizada de diversos modos para absorção de íons (Marenco \& Lopes, 2005).

Além de influenciar os processos de absorção, a temperatura também pode alterar a taxa de transporte de nutrientes, assim como a partição de fotoassimilados no floema. Segundo Taiz \& Zeiger (2004), o resfriamento de um tecido-dreno inibe as atividades que necessitam de energia metabólica, resultando na diminuição da velocidade do transporte em direção ao dreno. De acordo com Rena \& Maestri (1985), a temperatura ótima para assimilação de $\mathrm{CO}_{2}$ no cafeeiro varia de 20 a $30^{\circ} \mathrm{C}$, dependendo da temperatura em que as plantas foram aclimatadas nos dias anteriores. Deve-se salientar, entretanto que outros fatores, como disponibilidade hídrica, podem ter influenciado os resultados. Em janeiro de 2005 o índice pluvial foi de apenas $7 \mathrm{~mm}$ (Quadro 5) e o solo da altitude $720 \mathrm{~m}$ apresenta maior capacidade de retenção de água (Quadro 2).

As variações na concentração de $\mathrm{Ca}, \mathrm{Mg}$ e $\mathrm{S}$ no terceiro e no quarto par de folhas de ramos produtivos ao longo do ciclo reprodutivo do cafeeiro podem ser visualizadas na figura 2 e quadro 10. De modo geral, na altitude de $950 \mathrm{~m}$ a concentração foliar de nutrientes apresentou tendência de ser menor comparada à das demais altitudes, durante todo o período de formação dos frutos. É provável que as menores temperaturas ocorridas na altitude de $950 \mathrm{~m}$ tenham propiciado a ocorrência de menores concentrações, já que a temperatura possui efeito na absorção e distribuição de nutrientes (Taiz \& Zeiger, 2004; Marenco \& Lopes, 2005). No entanto, outros fatores, além da temperatura, podem ter atuado em conjunto na absorção e distribuição de nutrientes.

No quadro 11 pode-se observar a comparação entre as concentrações foliares de $\mathrm{Ca}, \mathrm{Mg}$ e S na época da floração (início do período reprodutivo) e as 

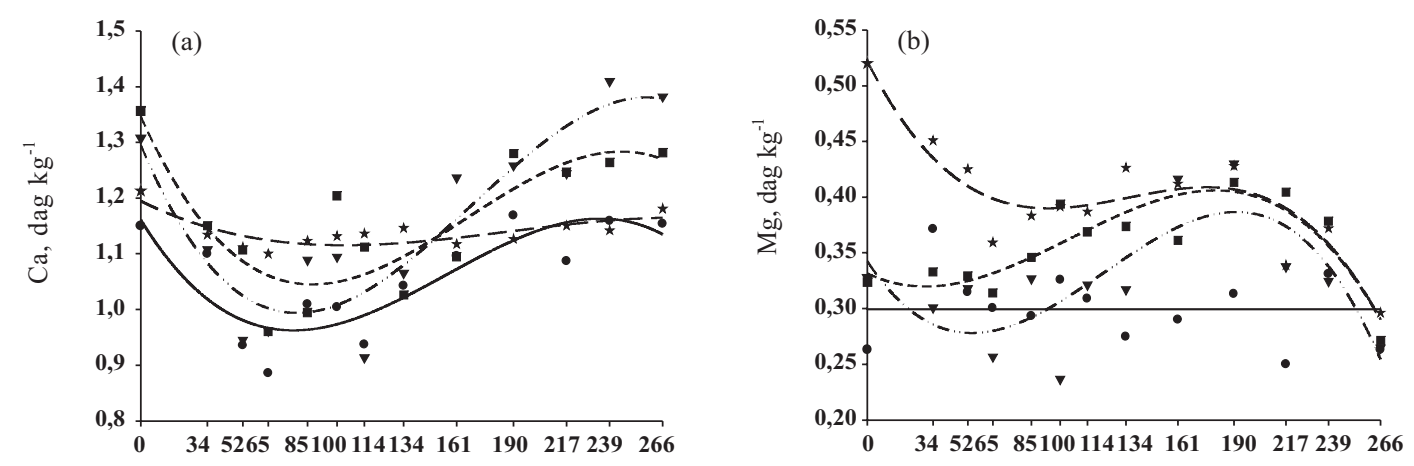

DIAS APÓS A ANTESE

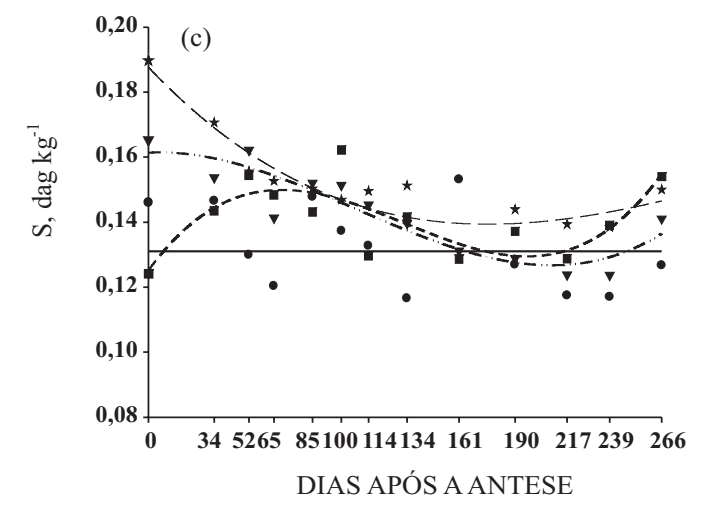

Figura 2. Concentração de $\mathrm{Ca}(\mathrm{a}), \mathrm{Mg}(\mathrm{b})$ e S (c) em folhas de cafeeiro em função do tempo decorrido após a antese, em quatro altitudes.

Quadro 10. Equações de regressão da variação nas concentrações de Ca, Mg e S em folhas de cafeeiros em função do tempo decorrido após a antese, em quatro altitudes, ponto de mínimo $\left(X_{\min }, D_{A A}\right)$ e máximo ( $\mathbf{X}_{\text {max }}$, DAA)

\begin{tabular}{|c|c|c|c|c|c|}
\hline Variável & Altitude & Modelo & $\mathbf{R}^{2}$ & $\mathbf{X}_{\min }$ & $\mathrm{X}_{\max }$ \\
\hline & $\mathrm{m}$ & & & & \\
\hline \multirow[t]{4}{*}{$\mathrm{Ca}$} & 720 & $\hat{y}=1,194-0,00175 x+0,0000116 x^{2}-0,0000000207 x^{3}$ & 0,635 & 104 & 0 \\
\hline & 800 & $\hat{y}=1,346-0,00782 x+0,0000607 x^{2}-0,000000121 x^{3}$ & 0,712 & 87 & 0 \\
\hline & 880 & $\hat{y}=1,295-0,00844 x+0,0000693 x^{2}-0,000000137 x^{3}$ & 0,834 & 80 & 258 \\
\hline & 950 & $\hat{y}=1,161-0,00574 x+0,0000491 x^{2}-0,000000104 x^{3}$ & 0,730 & 78 & 234 \\
\hline \multirow[t]{4}{*}{$\mathrm{Mg}$} & 720 & $\hat{y}=0,521-0,00341 \mathrm{x}+0,0000278 \mathrm{x}^{2}-0,0000000688 \mathrm{x}^{3}$ & 0,831 & 266 & 0 \\
\hline & 800 & $\hat{y}=0,331-0,000825 x+0,0000160 x^{2}-0,0000000507 x^{3}$ & 0,751 & 266 & 180 \\
\hline & 880 & $\hat{y}=0,342-0,00262 x+0,0000311 x^{2}-0,0000000844 x^{3}$ & 0,592 & 266 & 191 \\
\hline & 950 & $\hat{y}=\bar{y}=0,300$ & $\cdots$ & $-\cdot$ & -.- \\
\hline \multirow[t]{4}{*}{$\mathrm{S}$} & 720 & $\hat{y}=0,187-0,000626 \mathrm{x}+0,00000245 \mathrm{x}^{2}-0,00000000253 \mathrm{x}^{3}$ & 0,876 & 176 & 0 \\
\hline & 800 & $\hat{y}=0,125+0,000811 x-0,00000793 x^{2}+0,0000000199 x^{3}$ & 0,621 & 0 & 266 \\
\hline & 880 & $\hat{y}=0,161+0,0000300 \mathrm{x}-0,00000266 \mathrm{x}^{2}+0,00000000827 \mathrm{x}^{3}$ & 0,840 & 209 & 6 \\
\hline & 950 & $\hat{y}=\bar{y}=0,132$ & -.. & -.. &.- \\
\hline
\end{tabular}


Quadro 11. Concentrações foliares de $\mathrm{Ca}, \mathrm{Mg}$ e $\mathrm{S}$ na floração $\left(\mathrm{C}_{\mathrm{F}}\right)$ comparada às concentrações foliares no momento da TMAD dos elementos para os frutos nos estádios de expansão rápida $\left(\mathrm{C}_{\mathrm{ER}}\right)$ e granaçãomaturação $\left(\mathrm{C}_{\mathrm{GM}}\right)$, em quatro altitudes

\begin{tabular}{|c|c|c|c|c|c|c|}
\hline \multirow[t]{2}{*}{ Variável } & \multirow{2}{*}{$\frac{\text { Altitude }}{\mathrm{m}}$} & \multirow{2}{*}{$\begin{array}{l}\text { Florada } \\
\text { dag } \mathrm{kg}^{-1}\end{array}$} & \multicolumn{2}{|c|}{ Crescimento rápido } & \multicolumn{2}{|c|}{ Granação } \\
\hline & & & dag $\mathrm{kg}^{-1}$ & $\mathrm{C}_{\mathrm{ER}}-\mathrm{C}_{\mathrm{F}}$ & dag $\mathrm{kg}^{-1}$ & $\mathrm{C}_{\mathrm{GM}}-\mathrm{C}_{\mathrm{F}}$ \\
\hline \multirow[t]{4}{*}{$\mathrm{Ca}$} & 720 & 1,194 & 1,116 & $-0,078$ & 1,115 & $-0,079$ \\
\hline & 800 & 1,346 & 1,053 & $-0,293$ & 1,269 & $-0,077$ \\
\hline & 880 & 1,295 & 0,994 & $-0,301$ & 1,042 & $-0,253$ \\
\hline & 950 & 1,161 & 0,963 & $-0,198$ & 1,156 & $-0,005$ \\
\hline \multirow[t]{4}{*}{$\mathrm{Mg}$} & 720 & 0,521 & 0,394 & $-0,127$ & 0,393 & $-0,128$ \\
\hline & 800 & 0,331 & 0,334 & $+0,003$ & 0,393 & $+0,062$ \\
\hline & 880 & 0,342 & 0,281 & $-0,061$ & 0,351 & $+0,009$ \\
\hline & 950 & 0,300 & 0,300 & 0 & 0,300 & 0 \\
\hline \multirow[t]{4}{*}{$\mathrm{S}$} & 720 & 0,187 & 0,149 & $-0,038$ & 0,140 & $-0,047$ \\
\hline & 800 & 0,125 & 0,149 & $+0,024$ & 0,131 & $+0,006$ \\
\hline & 880 & 0,161 & 0,150 & $-0,011$ & 0,133 & $-0,028$ \\
\hline & 950 & 0,132 & 0,132 & 0 & 0,132 & 0 \\
\hline
\end{tabular}

concentrações foliares dos elementos nos respectivos momentos de máxima taxa de acúmulo diário (TMAD) para os frutos nos estádios de rápida expansão e granação. Observa-se que a concentração de Ca nas folhas diminuiu nos momentos das TMAD para o fruto, independentemente da altitude. Essas observações mostram que houve competição fruto/folha pela partição de Ca. Essa competição ocorreu pelo nutriente translocado no xilema, já que o $\mathrm{Ca}$ é um nutriente imóvel no floema (Marchsner, 1995; Epstein \& Bloom, 2006). Quanto a Mg e S, observou-se competição fruto/folha nos dois períodos de maior demanda pelo nutriente apenas na altitude de $720 \mathrm{~m}$. A maior velocidade de acúmulo nos frutos na menor altitude pode ter contribuído para que houvesse maior pressão de competição, já que os frutos são drenos prioritários (Rena \& Maestri, 1985).

\section{CONCLUSÕES}

1. A altitude teve influência na extensão do ciclo do cafeeiro, bem como no acúmulo de $\mathrm{Ca}, \mathrm{Mg}$ e $\mathrm{S}$ em frutos.

2. A TMAD (taxa máxima de acúmulo diário) no estádio de granação apresentou tendência de ser mais tardia com a elevação da altitude.

3. Independentemente da altitude, os maiores acúmulos relativos de $\mathrm{MS}, \mathrm{Ca}, \mathrm{Mg}$ e $\mathrm{S}$ foram observados no estádio de granação-maturação do fruto.
4. Na menor altitude, o acúmulo de Ca, Mg e S em frutos apresentou-se mais precoce.

5. De modo geral, na altitude de $720 \mathrm{~m}$ ocorreu maior competição fruto/folha pela partição de $\mathrm{Ca}, \mathrm{Mg}$ e S.

\section{AGRADECIMENTOS}

Ao Consórcio Nacional de Pesquisa e Desenvolvimento do Café (CNP\&D-Café) e ao Conselho Nacional de Desenvolvimento Científico e Tecnológico (CNPq) pelo apoio financeiro.

\section{LITERATURA CITADA}

ASSOCIATION OF OFFICIAL ANALYTICAL CHEMISTS AOAC. Official methods of analysis. 12.ed. Washington, 1975. $1094 \mathrm{p}$.

CAMARGO, A.P. As oito fases fenológicas da frutificação do cafeeiro. In: CONGRESSO BRASILEIRO DE PESQUISAS CAFEEIRAS, 24., Poços de Caldas, 1998. Anais. Rio de Janeiro, Instituto Brasileiro do Café, 1998. v.1. p.41-42.

CAMARGo, A.P. \& CAMARGO, M.B.P. Definição e esquematização das fases fenológicas do cafeeiro arábica nas condições tropicais do Brasil. Bragantia, 60:65-68, 2001.

CANNEL, M.G.R. Changes in the respiration and growth rates of developing fruits of Coffea arabica L. J. Hortic. Sci., 46:263-272, $1971 \mathrm{~b}$. 
CANNEL, M.G.R. Seasonal patterns of growth and development of arabica coffee in Kenya. Part IV. Effects of seasonal differences in rainfall on bean size. Kenya Coffee, 36:175-180, 1971a.

COOMBE, B.G. The development of fleshy fruits. Ann. Rev. Plant Physiol., 27:507-28, 1976.

EPSTEIN, E. \& BLOOM, A.J. Nutrição mineral de plantas: Princípios e perspectivas. 2.ed. Londrina, Planta, 2006. 401p.

GOUVEIA, N.M. Estudo da diferenciação e crescimento das gemas florais de Coffea arabica L.: Observação sobre antese e maturação dos frutos. Campinas, Universidade Estadual de Campinas, 1984. 237p. (Tese de Mestrado)

GUIMARÃES, P.T.G.; GARCIA, A.W.R.; ALVAREZ V., V.H.; PREZOTTI, L.C.; VIANA, A.S.; MIGUEL, A.E.; MALAVOLTA, E.; CORRÊA, J.B., LOPES, A.S.; NOGUEIRA, F.D. \& MONTEIRO, A.V.C. Cafeeiro. In: RIBEIRO, A.C.; GUIMARÃES, P.T.G. \& ALVAREZ V., V.H., eds. Recomendações para o uso de corretivos e fertilizantes em Minas Gerais, $5^{\mathrm{a}}$ Aproximação. Viçosa, MG, Comissão de Fertilidade do Solo do Estado de Minas Gerais - CFSEMG, 1999. p.289-302.

JACSON, M.L. Soil chemical analysis. New Jersey, Prentice Hall, 1958. 498p.

JOHNSON, C.M. \& ULRICH, A. Analytical methods for use in plants analyses. Los Angeles, University of California, 1959. v.766. p.32-33.

JONES JUNIOR, J.B.; WOLF, B. \& MILLS, H.A. Plant analysis handbook: a practical sampling, preparation, analysis, and interpretation guide. Athens, Micro-Macro Publishing, 1991. 213p.

LARCHER, W. Ecofisiologia vegetal. São Carlos, RiMa, 2004. $531 \mathrm{p}$.

LAVIOLA, B.G.; MARTINEZ, H.E.P.; SOUZA, R.G. \& ALVAREZ V., V.H. Dinâmica de cálcio em folhas e frutos de cafeeiro arábico em três níveis de adubação. R. Bras. Ci. Solo, 31:319-329, 2007.
LEON, J. \& FOURNIER, L. Crecimento y desarollo del fruto de Coffea arabica. Turrialba, 12:65-74, 1962.

MARENCO, R.A. \& LOPES, N.F. Fisiologia vegetal: Fotossíntese, respiração, relações hídricas e nutrição mineral. Viçosa, MG, Universidade Federal de Viçosa, 2005. 451p.

MARSCHNER, H. Mineral nutrition of higher plants. 2.ed. New York, Academic Press, 1995. 889p.

MATIELLO, J.B.; SANTIAGO, R.; GARCIA, A.W.R.; ALMEIDA, S.R. \& FERNANDES, D.R. Cultura de café no Brasil: Novo manual de recomendações. Rio de Janeiro, MAPA /PROCAFE, 2005. 438p.

MORAES, F.R.P. \& CATANI, R.A. A absorção de elementos minerais pelo fruto do cafeeiro durante o seu desenvolvimento. In: REUNIÃO ANUAL DA SBPC. Resumos das comunicações à XVI Reunião Anual da SBPC. Ci.Cult., 16:142, 1964.

PUSCHMANN, R. Características bioquímicas do fruto do cafeeiro (Coffea arabica L.) durante a maturação. Viçosa, MG, Universidade Federal de Viçosa, 1975. 35p. (Tese de Mestrado)

RAMÍREZ, F.; BERTSCH, F. \& MORA, L. Consumo de nutrimentos por los frutos y bandolas de cafe Caturra durante um ciclo de desarrollo y maduracion en Aquiares, Turrialba, Costa Rica. Agron. Costarricence, 26:33-42, 2002.

RENA, A.B. \& MAESTRI, M. Fisiologia do cafeeiro. Inf. Agropec., 11:26-40, 1985.

RENA, A.B.; BARROS, R.S. \& MAESTRI, M. Desenvolvimento reprodutivo do cafeeiro. In: ZAMBOLIM, L. Tecnologias de produção de café com qualidade. Viçosa, MG, Universidade Federal de Viçosa, 2001. p.101-128.

TAIZ, L. \& ZEIGER, E. Fisiologia vegetal. 3.ed. Porto Alegre, Artmed, 2004.719p.

VENEGAS, J.G.; HARRIS, R.S. \& SIMON, B.A. A comprehensive equation for the pulmonary pressure. Volume curve. J. Appl. Physiol., 84:389-395, 1998. 